\title{
Single-Walled Nanohorn
}

National Cancer Institute

\section{Source}

National Cancer Institute. Single-Walled Nanohorn. NCI Thesaurus. Code C62387.

A carbon nanotube that is comprised of a single layer of graphitic carbon and exhibits an irregular horn-like shape. One of the primary characteristics of carbon nanohorns is their ability to agg regate. This aggregation is advantageous because of the resulting enhanced permeability in both gases and liquids. 